\title{
Severe thrombocytopenia soon after drug-eluting stent implantation in ST-elevation myocardial infarction
}

\author{
Agnieszka Łebek-Szatańska, Anna Pośnik-Kisło, Wiesława Błaszak-Ciećwierska, Marek Dąbrowski \\ Cardiology Clinical, Physiotherapy Division, $2^{\text {nd }}$ Faculty of Medicine, Medical University of Warsaw, Bielański Hospital, Warsaw, Poland \\ Postep Kardiol Inter 2015; 11, 4 (42): 354-356 \\ DOI: $10.5114 /$ pwki.2015.55614
}

\begin{abstract}
A bstract
Thrombocytopenia is among the most frequent haematological issues in patients hospitalised with myocardial infarction. We discuss the case of a 77-year-old male who was admitted to hospital on the $4^{\text {th }}$ hour of ST-elevation myocardial infarction of the anterior wall. A percutaneous coronary intervention to critically stenoted initial segment of the anterior descending branch was performed. A few days later he started developing symptomatic thrombocytopenia. On the course of his long hospitalization we faced many important problems, concerning both diagnosis and treatment of these co-existing conditions.
\end{abstract}

Key words: myocardial infarction, coronary angioplasty, trombocytopenia.

\section{Introduction}

According to literature data, thrombocytopenia is diagnosed in even over a dozen percent of patients hospitalized because of an acute coronary incident. Its occurrence increases the risk for both thrombotic and bleeding complications, influencing short- and long-term prognosis. In the subgroup of patients with ST-elevation myocardial infarction (STEMI) treated with primary coronary angioplasty the condition occurs in at most several percent of patients. In these cases, there is still a lot of controversy regarding treatment [1-3].

This report describes the case of a 77-year-old male patient with STEMI who developed a significant drop in platelet count in the early period after drug-eluting stent implantation. We discuss problems that we encountered, regarding diagnosis as well as pharmacotherapy of these coexisting diseases.

\section{Case report}

A 77-year-old male patient was admitted to the Cardiology Department in the $4^{\text {th }}$ hour of chest pain while at rest. Two days earlier the patient had been discharged from the General Surgery Ward, following an approximately 3-week long hospitalization because of incarcerated inguinal hernia with segmental necrosis of the small intestine. At that time signs of heart failure with low ejection fraction had been observed and confirmed with echocardiographic investigation. Laboratory tests of the clotting system and blood cell count revealed no abnormalities. Haemostasis disorders had not been found during any of surgical procedures. History of bleeding or bleeding tendencies was negative, and family history was unremarkable. From the admission day to the Surgery Department the patient received low molecular weight heparin in a preventive dose, which was continued at home. At admission to the Cardiology Department, the diagnosis of STEMI of the anterior wall was made and angioplasty of the critically stenotic anterior descending artery with implantation of an everolimus-eluting stent was performed. A standard dose of 5000 IU of unfractionated heparin was administered intravenously during the procedure, with no glycoprotein IIb/IIla receptor inhibitor (because of recent abdominal surgery). Aspirin and clopidogrel were introduced in recommended saturating doses and enoxaparin was discontinued. At admission the platelet count was 124 000/ $\mu$ l. Control blood cell counts performed throughout the hospitalization revealed a gradual reduction of the platelet count to $57,000 / \mu$ l on day 4 , and to $4,000 / \mu$ l on day 8 after the angioplasty (verified in citrate-collected blood). On day 10 dot-like petechiae occurred in the area of the sternum, feet and lower legs. Laboratory data confirmed normal clotting parameters, blood cell count, haemoglobin level,

\section{Corresponding author:}

Agnieszka Łebek-Szatańska, Cardiology Clinical, Physiotherapy Division, $2^{\text {nd }}$ Faculty of Medicine, Medical University of Warsaw, Bielański Hospital, 9/274 Zgrupowania Żmija St, 01-875 Warsaw, Poland, fax: +48 694540 351, e-mail: agalebek@gmail.com Received: 14.02.2015, accepted: 26.03.2015. 
and hepatic and renal function indicators. We were not able to perform any specialist haematological tests in our laboratory.

The patient received empirically intravenous pulses of methylprednisolone, and after that oral glucocorticosteroid therapy with prednisone was continued. Considering the platelet count remaining at the level of several thousand per $\mu \mathrm{l}, 100 \mathrm{ml}$ ( 2 units) of platelet concentrate were transfused, achieving an increase of the platelet count of up to $13,000 / \mu \mathrm{l}$. Moreover, fondaparinux was introduced at the preventive dose of $2.5 \mathrm{mg} /$ day, along with ticagrelor (instead of aspirin and clopidogrel) at the dose of $90 \mathrm{mg}$ bid. As a gradual increase of the platelet count was observed, the steroid dose was down-titrated. Finally, the patient was discharged on the $50^{\text {th }}$ day of hospitalisation, with a platelet count of $200,000 / \mu$ l. At the patient's request, clopidogrel was reinstated for financial reasons, before the dismissal.

\section{Discussion}

One of the most important problems that has to be solved at the very beginning of treatment of a patient with STEMI and low platelet count is continuation or cessation of antiplatelet therapy. Aspirin discontinuation is justified by an increased risk of gastrointestinal bleeding. On the other hand, clopidogrel discontinuation is suggested by the risk of thrombotic thrombocytopenic purpura (TTP), and also of trilineage pancytopenia in rare cases $[1,4]$. The pathogenesis of TTP stems from a severe deficiency of the enzyme ADAMTS13 or from its inhibition, which can be used in diagnosis. It usually occurs 10-15 days after treatment with thienopyridines. Ticagrelor was proved more advantageous and as safe as clopidogrel in the PLATO study [5]. As a result, current guidelines of STEMI treatment recommend first line ticagrelor in all patients subject to angioplasty, and the use of clopidogrel is recommended only in those situations when other drugs are unavailable [6]. Unfortunately, the cost of therapy is still a significant barrier in Poland.

Diagnosis of the type of thrombocytopenia was another issue in the discussed case. The clinical course suggested the immunological mechanism of the disease. Heparin-induced thrombocytopenia and idiopathic thrombocytopenia considered by us are diagnostically challenging conditions. Both cannot be fully confirmed using objective laboratory tests. Therefore, this is not the first published case where those types of thrombocytopenia are considered simultaneously or in sequence [7-9].

Heparin-induced thrombocytopenia (HIT) is a specific type of drug-induced reaction, in which the pathological process consists in heparin-dependent formation of IgG antibodies against neoepitopes in platelet factor 4 (PF 4). However, detection of those antibodies is not synonymous with the diagnosis of HIT, because HIT symptoms develop in only a low percentage of individuals with a positive titre of them (the "iceberg model"). Because of that, and because of poor availability of tests and delayed results, in Poland HIT is most often diagnosed based on the characteristic clinical presentation. In the case discussed, the only considered type of heparin-dependent reaction was the so-called delayed-onset HIT, occurring in as few as $3-5 \%$ of all cases [10]. In that form of the disease, antibodies produced with participation of heparin may activate platelets also without its presence in the circulation, imitating an autoimmune disease [11]. Thus, thrombocytopenia develops already after discontinuation of heparin. Clinically, that form of HIT may be non-specifically manifested by a reduced platelet count to a value below 20,000/ $\mu$ l, a tendency for bleeding as well as a slow return of the platelet count to normal [10].

In the case of non-available appropriate laboratory testing, it is recommended to use different prognostic scores to evaluate the risk of HIT in everyday clinical practice. The most thoroughly evaluated clinical scoring system is the 4Ts score (Thrombocytopenia, Timing, Thrombosis and oThers). Although the score is considered to have a high negative predictive value (in the case of a 4Ts score below 4), its positive predictive value varies greatly depending on the experience of the physician applying the score [11]. What is more, the score is not as useful in situations where untypical types of HIT are suspected. In the case discussed above, the patient's 4Ts score was 4, indicating that HIT should be still considered as a potential cause of thrombocytopenia. In this situation, the HIT antigen assay (platelet factor 4/polyanion immunoassay) or platelet activation assays (for example, platelet serotonin release assay (14C-SRA) or heparin-induced platelet activation (HIPA) assay), based on which HIT could be ruled out, should be performed, especially considering that the prevention of thrombosis in the recently implanted stent was an issue of great concern.

Fondaparinux used by us is regarded as safe and effective HIT therapy, and some early concerns regarding possible induction of fondaparinux-dependent thrombocytopenia - by similarity to heparin - have not been confirmed [11, 12]. The advantage of the drug is a possibility of using two dosages - therapeutic and prophylactic. In the face of existing diagnostic difficulties and literature-reported overdiagnosis of HIT, application of a prophylactic dose seems justified in that case, because the probability of HIT was not high. That regimen is a continued anti-thrombotic prevention with a drug alternative to heparin, rather than an actual HIT therapy [10].

In cases when HIT diagnosis is possible, platelet concentrate transfusions should be used with extreme caution, for the procedure may intensify the ongoing processes, increasing both the risk of thrombosis and bleeding ("adding fuel to the fire") [10].

In the situation that we could not exclude heparin-induced thrombocytopenia, the clinical course of the discussed patient might also suggest the diagnosis of 
primary immunological thrombocytopenia (ITP). However, the lack of ITP events in the past history make this diagnosis less probable. The ITP is an acquired chronic condition of unclear aetiology, affecting mainly women aged 20 to 50 years. Its diagnosis is principally a negative one. It is associated with stimulation of production of IgG antibodies against platelet glycoproteins. However, those antibodies are detected in approximately $60 \%$ of patients, and hence cannot be a basis for the diagnosis. The response to steroids, the essential therapy in ITP, is usually seen in both ITP and HIT.

\section{Conclusions}

To summarise, the example of our patient may illustrate the "kaleidoscope of immunity" phenomenon [13], where two or more autoimmune diseases co-exist in one patient - one after another or as overlap syndromes. However, according to data this problem is rather casuistic if concerning thrombocytopenic conditions [7, 8]. On the other hand, the course of hospitalization of our patient and the difficulties in management were also due to still imperfect diagnostic methods and limitations in their availability, as well as in cooperation with other specialists, including a haematologist, even in clinical hospitals.

\section{Conflict of interest}

The authors declare no conflict of interest.

\section{References}

1. Dziewierz A, Dudek D. Symposium: How to manage patients with acute coronary syndrome and comorbidities. How to manage a patient with acute coronary syndrome and concomitant hematological disorders - viewpoint of an invasive cardiologist. Postep Kardiol Inter 2008; 4: 164-72.

2. Wang TY, Ou FS, Roe MT, et al. Incidence and prognostic significance of thrombocytopenia developed during acute coronary syndrome in contemporary clinical practice. Circulation 2009; 119: 2454-62.

3. Caixeta A, Dangas GD, Mehran R, et al. Incidence and clinical consequences of acquired thrombocytopenia after antithrombotic therapies in patients with acute coronary syndromes: results from the Acute Catheterization and Urgent Intervention Triage Strategy (ACUITY) trial. Am Heart J 2011; 161: 298-306.

4. Wojnar J. Leczenie krwawień u chorych z ostrymi zespołami wieńcowymi - problem interdyscyplinarny. Uwagi hematologa. Kardiochir Torakochir Pol 2011; 8: 472-4.

5. Wallentin L, Becker RC, Budaj A, et al. Ticagrelor versus clopidogrel in patients with acute coronary syndrome. N Engl J Med 2009; 361: 1045-57.

6. Task Force on the management of ST-segment elevation acute myocardial infarction of the European Society of Cardiology (ESC). Steg PG, James SK, Atar D, et al. ESC Guidelines for the management of acute myocardial infarction in patients presenting with ST-segment elevation. Eur Heart J 2012; 33: 2569-619.

7. Waheed F, Naseer N, Ahmed T, et al. Two patients with heparin-induced thrombocytopenia followed by idiopathic (immune) thrombocytopenic purpura: case report. Am J Hematol 2003; 73: 290-3.
8. Kravitz MS, Shoenfeld Y. Thrombocytopenic conditions-autoimmunity and hypercoagulability: commonalities and differences in ITP, TTP, HIT, and APS. Am J Hematol 2005; 80: 232-42.

9. Salloum R, Liu CY, Weise AM. Possible case of levofloxacin-induced thrombocytopenia. Am J Health Syst Pharm 2011; 68: 43-6.

10. Warkentin TE. HITlights: a career perspective on heparin-induced thrombocytopenia. Am J Hematol 2012; 87 Suppl. 1: S92-9.

11. Bakchoul T, Greinacher A. Recent advances in the diagnosis and treatment of heparin-induced thrombocytopenia. Ther Adv Hematol 2012; 3: 237-51.

12. Kumar M, Abrina VM, Chittimireddy S. Pulmonary embolism caused by delayed heparin-induced thrombocytopenia in a patient who received prophylactic LMWH. Am J Case Rep 2012; 13: 118-21.

13. Anaya JM, Corena R, Castiblanco J, et al. The kaleidoscope of autoimmunity: multiple autoimmune syndromes and familial autoimmunity. Expert Rev Clin Immunol 2007; 3: 623-35. 